\title{
REVIEW
}

\section{Clinical Application of 4D Flow MR Imaging for the Abdominal Aorta}

\author{
Yasuo Takehara ${ }^{1^{*}}$
}

\begin{abstract}
Blood vessels can be regarded as autonomous organs. The endothelial cells on the vessel surface serve as mechanosensors or mechanoreceptors for the flow velocity and turbulence of the blood flow in terms of wall shear stress (WSS), thereby monitoring changes in the flow velocity. Accordingly, the endothelial cells regulate the flow velocity by releasing numerous mediators. Such regulatory systems also trigger atherosclerosis, where the WSS decreases or fluctuates to maintain the flow velocity or local WSS. As occurrences of abdominal aortic aneurysms and aortic dissection are closely related to atherosclerosis, understanding the hemodynamics of the abdominal aorta is necessary to obtain useful information concerning the pathogenesis, diagnosis, and interventions. 4D flow MRI is beneficial for measuring the hemodynamics through comprehensive retrospective flowmetry of the entire spatio-temporal distributions of the flow vectors. This section focuses on abdominal aortic aneurysms and aortic dissection as representative examples of abdominal aortic diseases. Their hemodynamic characteristics and how hemodynamics is involved in their progression are described, and how 4D flow MRI can contribute to their assessment is also explained.
\end{abstract}

Keywords: abdominal aortic aneurysm, 4D flow magnetic resonance imaging, oscillatory shear index, phase-contrast image, wall shear stress

\section{Aging Aorta}

The aorta is not only a conduit that conducts blood from the heart to the peripheral circulation but also an autonomous organ. Autonomy means that if the blood flow through the lumen decreases, the vessel will contract to maintain the flow velocity and volume. Meanwhile, the blood vessel will dilate to reduce the flow velocity if it is higher than that required by the organs. The endothelial cells on the vessel surface serve as mechanosensors or mechanoreceptors for flow velocity and turbulence of the blood flow in terms of the wall shear stress (WSS) (Fig. 1a and 1b) and its fluctuations with the luminal flow. When the WSS declines and oscillates, the endothelial cells decrease the release of nitric oxide (NO) and contract the vessels to maintain the flow velocity. In the medium to long term, such control is dependent on permanent pathological changes in the wall. The

${ }^{1}$ Departments of Fundamental Development for Low Invasive Diagnostic Imaging and Radiology, Nagoya University Graduate School of Medicine, Nagoya, Aichi, Japan

*Corresponding author: Department of Fundamental Development for Advanced Low Invasive Diagnostic Imaging Nagoya University, Graduate School of Medicine, 65, Tsurumai-cho, Showa-ku, Nagoya, Aichi 466-8550, Japan. Email: takehara@med.nagoya-u.ac.jp

This work is licensed under a Creative Commons Attribution-NonCommercial-NoDerivatives International License.

(C2022 Japanese Society for Magnetic Resonance in Medicine

Received: December 5, 2021 | Accepted: January 5, 2022 endothelial cells control the coagulation and fibrinolysis system via the prostaglandin $\mathrm{I}_{2}\left(\mathrm{PGI}_{2}\right)$ tissue plasminogen activator (t-PA) or thrombomodulin (TM), as well as the inflammatory system via vascular cell adhesion molecule-1 (VCAM1), etc., thereby promoting atherosclerosis for narrowing the lumen (Fig. 2). ${ }^{1-4}$ Furthermore, the aorta is prone to age-related degeneration of the vascular endothelium and media. The elastin in the media is replaced by collagen, and the vessel wall stiffness increases. Thus, the effects of arterial stiffness are initially observed as an increase in pulse wave conduction velocity (PWV). ${ }^{5}$ The wall stiffness leads to remodeling of the aortic wall, which manifest as aortic lengthening and the resultant tortuosity. ${ }^{6}$ The response is reasonable when maintaining the flow velocity to the vital organs; however, it may induce lifethreatening vascular diseases such as the rupture of aneurysms or aortic dissections.

\section{Atherosclerosis Research Using 4D Flow MRI}

The inference that pathological changes in the blood flow may lead to arterial lesions has long been reported, and researchers have provided substantial evidence that the vascular endothelium has receptors for flow velocity based on the WSS and its fluctuations, where the oscillatory shear (OS) is simulated by the OS index (OSI). ${ }^{7}$ As this fact has become common knowledge in the field 
a

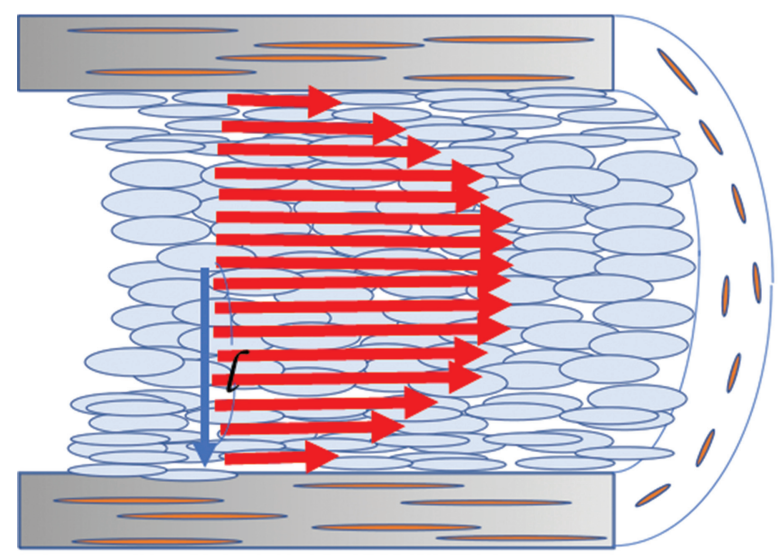

Wall Shear Stress $>1.5 \mathrm{~Pa}$ b

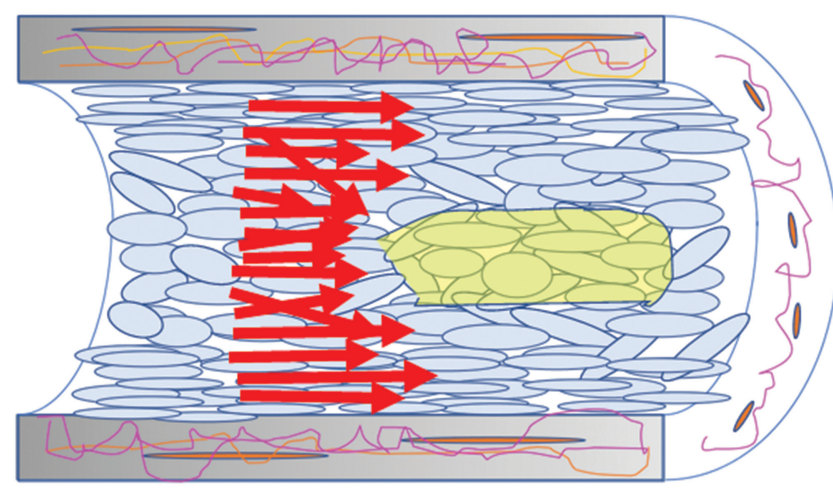

Wall Shear Stress $<1.5 \mathrm{~Pa}$

Fig. 1 a: Schematic of the healthy arterial wall in a state of normal laminar blood flow. Velocities of the laminar flow distribute a high-velocity stream in the center of the vessel lumen and a low-velocity stream near the wall owing to the WSS (red arrows), which can be regarded as the frictional force of the blood flow. The WSS is calculated by differentiating the velocity of the blood flow normal to the vessel wall and multiplying the result by the blood viscosity: $(\mu \cdot \mathrm{dv} / \mathrm{dl})$. There are mechanosensors for the WSS on the inner surface of the vascular endothelium, and in the normal hemodynamic status of the laminar flow, the endothelium is aligned with the flow. The red arrows represent the flow velocity vectors. $\mathbf{b}$ : When blood flow becomes slow and fluctuates (red arrows), the WSS decreases. The endothelial cells are not aligned along the blood stream in such hemodynamics. Decreased WSS $<1.5$ Pa and increased oscillatory shear stress induce arterial contraction and trigger an atherogenic process in the vascular intima and media. Collagenous fibers (pink lines) are induced in the media. The yellow areas represent plaque that has begun to form under the endothelium. WSS, wall shear stress.

of vascular physiology, attempts have been made to examine the human WSS of the aorta in vivo from MR images using 2D phase-contrast methods, etc. ${ }^{8}$ As the aorta is a relatively large organ with a sizeable luminal volume and is located over a geometrically wide range in the body, i.e., from the chest to the abdomen, 2D methods are not suitable for examining it. Therefore, such studies are usually conducted via computer simulation (computational fluid dynamics [CFD]). Using the conventional 2D MR method, it is possible to combine $2 \mathrm{D}$ data to reconstruct $3 \mathrm{D}$ data with a long imaging time; however, this method was not clinically viable until the advent of 4D flow MRI. As 4D flow MRI can measure all the velocity vectors in the ROI en-bloc, it is suitable for measuring and displaying such WSS in a $3 \mathrm{D}$ manner.

Owing to its comprehensive coverage of the aorta and hemodynamic information, 4D flow MRI can be used to measure the pulse wave propagation velocity, which is an indicator of arterial wall stiffness and is associated with higher prevalence of plaque. $^{9}$ The capability of $4 \mathrm{D}$ flow MRI in measuring the PWV is noteworthy because the PWV is associated with a higher incidence of cardiovascular events. ${ }^{9-11}$

A new hemodynamic derivative has recently attracted considerable research attention. ${ }^{12,13}$ As 4D flow MRI can measure the spatio-temporal velocity field in the entire volume of interest, indices reflecting the dissipation of viscous energy such as energy loss (EL) can also be measured using equations based on spatiotemporal velocity data. ${ }^{13-15}$ EL is the energy loss due to friction between viscous blood and the vessel wall or between blood. Previously, researchers used this indicator as a virtual catheter to fascilitate reproducible automated evaluation of volumetric intra-aortic hemodynamics alterations observed in the bicuspid aortic valve. ${ }^{16,17}$

Calculation of the EL using the turbulent kinetic energy (TKE) has also been reported. The TKE describes the fluctuation in kinetic energy during turbulence, which indicates the severity of diseases causing jet flow. This value is higher in areas with a non-laminar flow with high Reynolds number. ${ }^{18}$

\section{Development of Abdominal Aortic Aneurysm}

Abnormal aortic blood flow and aortic remodeling develop with a close interaction. A typical example is the development of an abdominal aortic aneurysm (AAA). Atherosclerosis is considered to be the leading cause of the development of AAAs. Several structural changes in the arterial wall, including the thinning, splitting, fraying, fragmentation, and loss of orderly arrangement of elastin fibers and elastic laminae, have been reported with aging. ${ }^{19}$ These changes could, in turn, induce dilation, stiffening, thickening, and elongation of the artery attributable to 


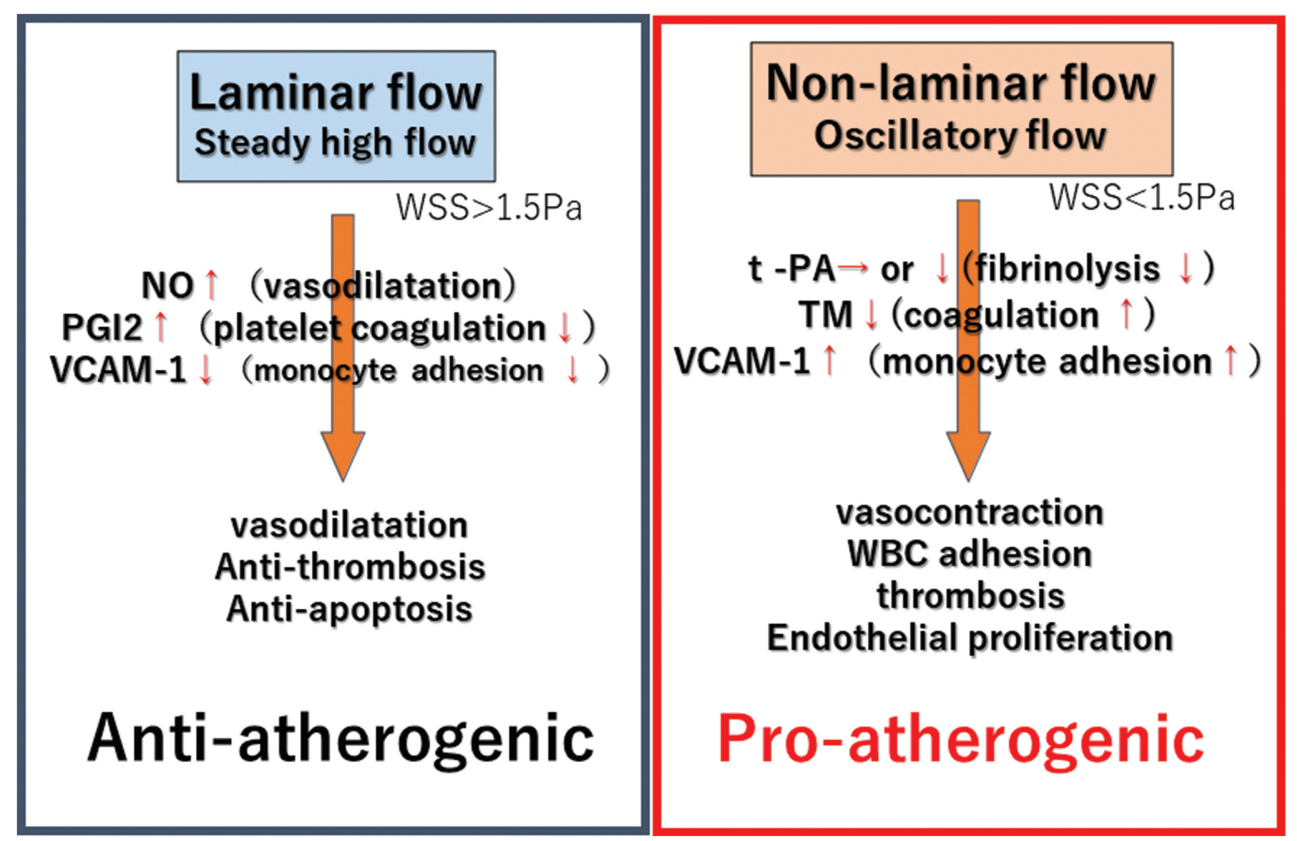

Fig. 2 The vascular endothelium constantly monitors the WSS. When the WSS decreases or fluctuates, it decreases the release of nitric oxide to increase the flow velocity by contracting the vessel wall. As the decrease in the WSS continues, the atherogenic process is triggered, thereby inducing permanent luminal narrowing. Therefore, to maintain a healthy vessel wall, the flow pattern must be laminar, and the flow velocity must be held at a certain high level. WSS, wall shear stress. (Reused from reference \#3)

fibrous remodeling. ${ }^{20,21}$ In general, AAAs are most commonly found in the terminal portion of the aorta, just above the bifurcation. Although the reason for this fact has not been clearly established thus far, it may be related to the infrarenal abdominal aorta, i.e., in a sense, the terminal portion of the flow path distal to the heart, where a steady laminar flow is extremely difficult to maintain under pulsatile cardiac pumping. This portion is constantly exposed to reflected waves from the lower extremities at end-systole. Typically, these reflected pulse waves are absorbed by the compliance of the aorta (Windkessel effect), and the collision of the downstream and upstream waves is alleviated. However, as the rigidity of the aortic wall increases, the wall compliance decreases and the flow of the reflected wave cannot be smoothly absorbed. Furthermore, when the PWV increases owing to arterial stiffness, turbulence dominates the aortic end portion owing to further violent collisions between the forward-flowing high-speed blood flow upstream and the reflected waves from the lower limbs. At the same time, the progression of arteriosclerosis also leads to flow channel deformities, which also begin to affect the hemodynamics. When the aorta loses its elasticity, it becomes elongated or over-lengthened, leading to aortic tortuosity. ${ }^{22}$ Aortic tortuosity results in flow detachment, and the detached flow decreases the local WSS. The vascular endothelium promotes an inflammatory process by stimulating macrophage induction to the subendothelial layers. Macrophages phagocytose cholesterol associated with modified low-density lipoprotein, eventually becoming foam cells. Then, plaque is formed on the aortic wall and promotes ulcers in the arterial surface, disrupting local blood flow. As the blood flow in the lumen of the vessel becomes non-laminar, the WSS fluctuates and decreases. These conditions further promote the wall fragility induced by atherosclerosis.

\section{Accelerated Growth of the AAA}

The risk of rupture of the aortic wall increases when the internal diameter of the aorta exceeds a specific range (i.e., 40-50 $\mathrm{mm}){ }^{23}$ Therefore, surgical intervention is generally indicated for AAAs exceeding $50 \mathrm{~mm}^{24}$ When dilation occurs in the abdominal aorta, the blood flow velocity in the lumen of the dilated segment decreases and becomes non-laminar compared to the upstream non-dilated segment (Fig. 3). This process leads to a further reduction in WSS and an increase in OSI in the dilated part, and vascular endothelial cells detect the signals and then induce the atherogenic processes of the aortic wall via the release of pro-atherogenic substances. 4D flow MRI allows in vivo measurement of the decreased WSS and increased OSI in the dilated segment of the aorta. In addition, the trend is proportional to the increase in the maximum internal diameter of the dilated component (Figs. 3, 4, and 5). ${ }^{25}$

Together with the pro-atherogenic changes in the WSS and OSI, the EL also increases around the aneurysmal neck (Fig. 6), reflecting the generation of non-laminar flows, such as vortex, helical, and turbulent flows (Fig. 3). Physical and mechanical changes also occur in the dilated portion of the 


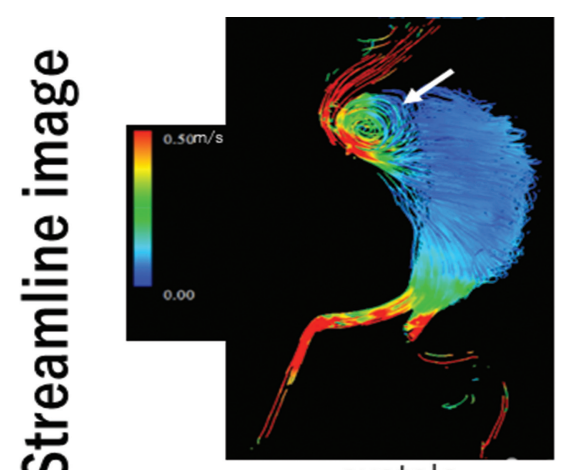

systole

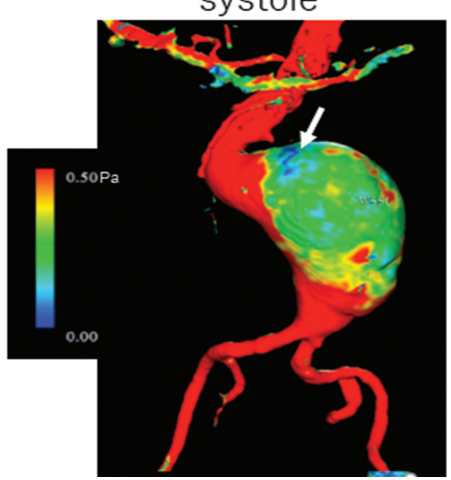

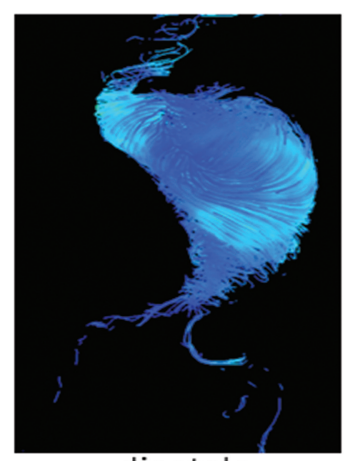

diastole

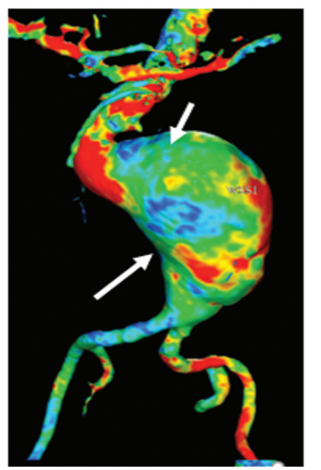

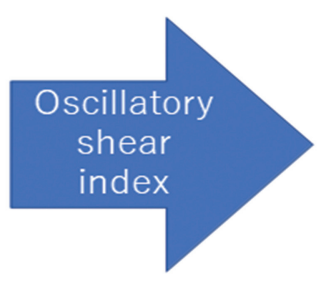

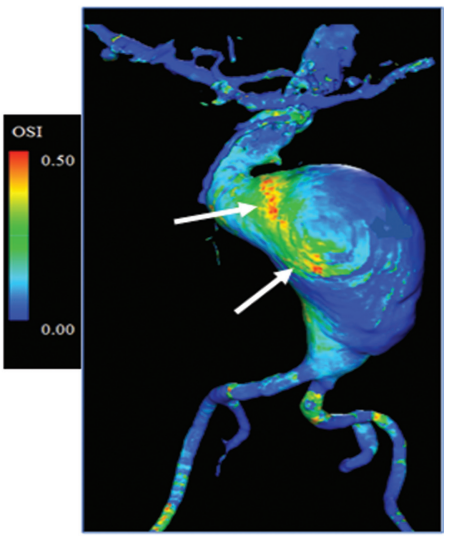

Fig. 3 Various hemodynamic assessments using 4D flow MRI for a male patient in his 80s with abdominal aortic aneurysm. Upper row: streamline images of the systole and diastole. Note a vortex flow (arrow) in the abdominal aortic aneurysm, particularly near the aneurysmal neck. High velocity is coded red. Lower row: WSS images at the systole and diastole. The WSS of the dilated aneurysmal portion is at an all-time low. Some portions (arrows) have a particularly low WSS. Low WSS is coded blue, and high WSS is coded red.The right column shows the OSI derived from temporal changes in the local WSS. Fluctuating WSS (high OSI) is coded red (arrows). OSI, oscillatory shear index; WSS, wall shear stress.

aorta when the blood flow becomes non-laminar with reduced velocity; the static pressure increases in the dilated segment owing to the law of energy conservation. ${ }^{26,27}$ The aortic wall, which has become vulnerable due to atherosclerosis, yields to the increased static pressure and protrudes further (Fig. 7). When aortic deformity occurs, the blood flow pattern in the dilated lumen becomes even more chaotic, ${ }^{25}$ which stimulates the endothelial cells to release pro-coagulation and anti-fibrinolytic mediators, leading to mural thrombus formation. The formation of a mural thrombus prevents oxygen diffusion from the blood to the wall and sometimes occludes the vasa-vasorum to make the vessel wall further vulnerable. Thus, this vicious cycle continues at an accelerated rate (Fig. 8).

\section{Interventional Treatment of AAA and 4D Flow MRI}

To prevent the rupture of AAAs, abdominal aortic graft replacement is performed for individuals at a high risk of rupture. Endovascular aortic repair (EVAR) is the procedure of choice if open surgery is not indicated. To date, there is no coherent report on the local hemodynamic changes occurring before and after EVAR.
However, the velocity of blood flow through the stentgraft lumen inevitably increases because of the decrease in the luminal diameter for the stent graft compared to the pre-EVAR AAA (Fig. 9). In addition, the compliance of the aorta with the stent-graft placement decreases after EVAR; therefore, the reflectional flow from the bilateral iliac arteries cannot be alleviated. There may be a concern that viscous dissipation in the stent may lead to an increased EL under these ciucumstances. The hemodynamic difference between artificial grafts used for open surgery and stent-grafts used for EVAR, as well as their effects on cardiac afterload require further clarification.

\section{Assessment of Type II Endoleak Using 4D Flow MRI}

One of the main drawbacks of EVAR is endoleak. An endoleak is a complication that causes a post-EVAR AAA to re-grow into an aneurysmal sac, and, in the worst case, it leads to rupture of the AAA. 4D flow MRI may be useful for predicting the re-growth of AAA due to type II endoleak after EVAR. ${ }^{28}$

4D flow MRI can simultaneously measure the blood flow velocity of multiple tributary arteries. The velocity and flow 


\section{Spatially-averaged WSS vs. Cardiac Phase}

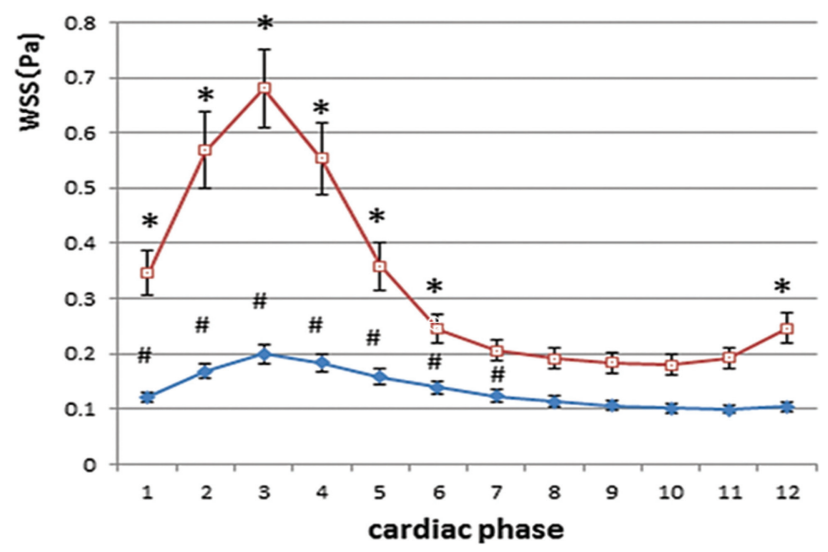

The Diameter Ratio vs. Amplitude of the Spatially Averaged WSS of the Dilated Aorta
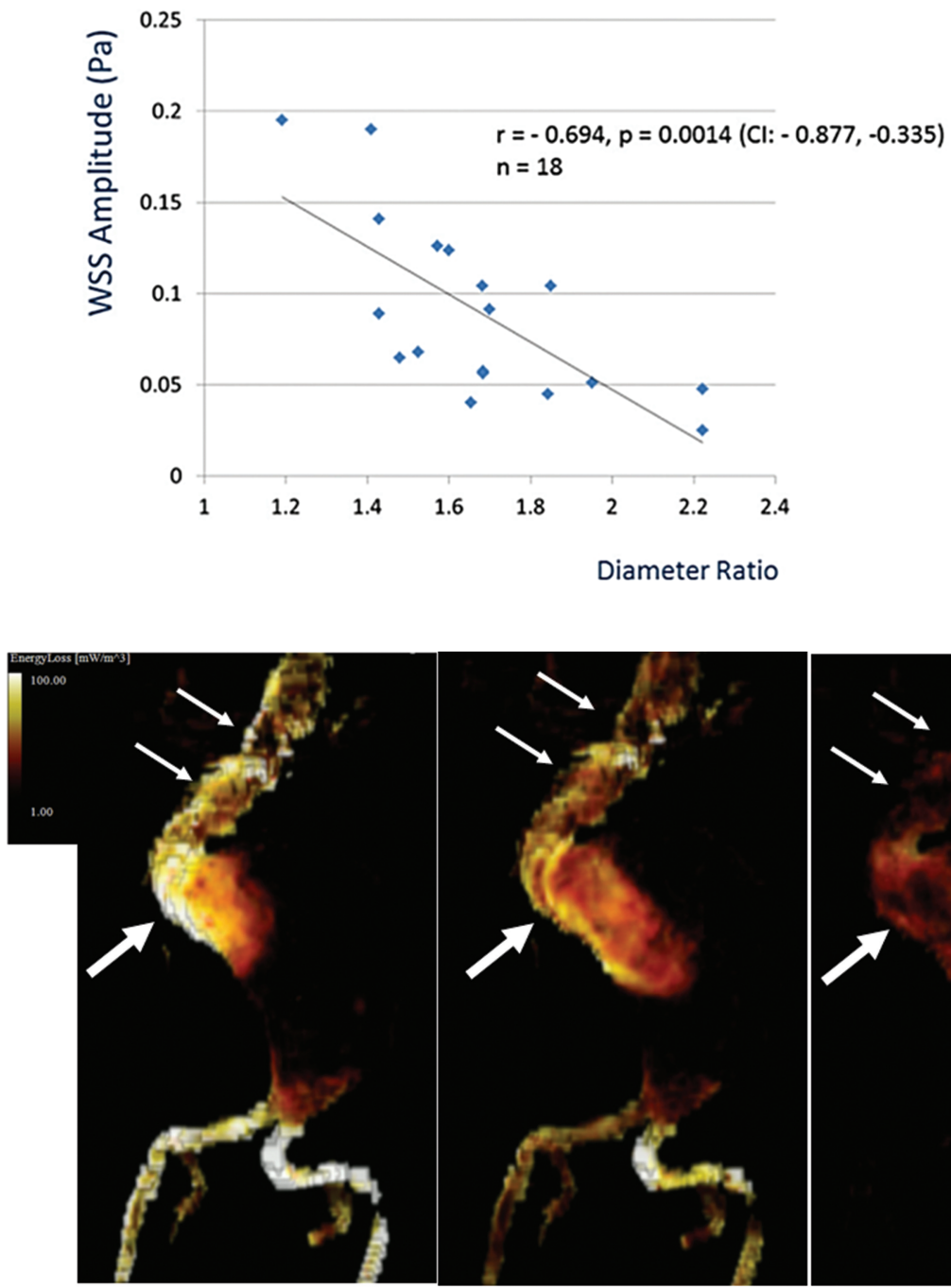

mid systole

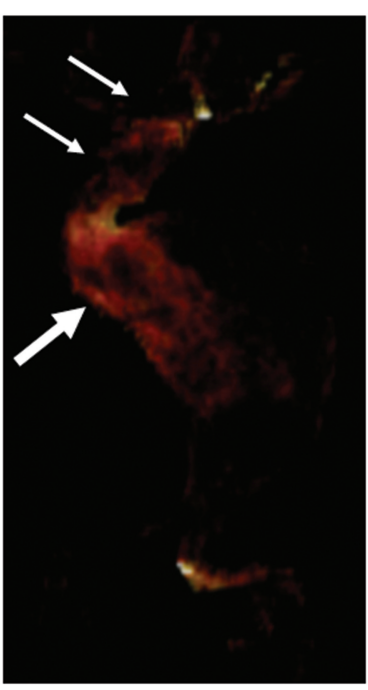

mid diastole
Fig. 4 Spatially averaged WSS of the abdominal aortic dilation is lower at all cardiac phases as compared to immediate upstream nondilated segment. "Spatially averaged" means WSS/area of the ROI. WSS, wall shear stress. (Reused from reference \#46)

Fig. 5 The amplitude of the spatially averaged WSS of the abdominal aortic aneurysm during the cardiac cycle is inversely proportional to the diameter ratio (maximum diameter of the aneurysm/ maximum diameter of the immediate undilated upstream segment). WSS, wall shear stress. (Reused from reference \#46)

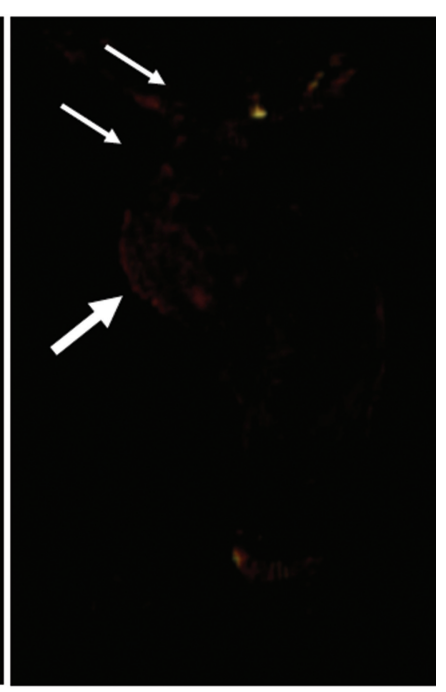

end diastole

Fig. 6 The EL was displayed on the MR angiogram of the same patient shown in Fig. 3. High-velocity flow in the undilated segment (small arrows) and vortex flow (large arrow) after kinked aorta results in higher EL. EL, energy loss. 


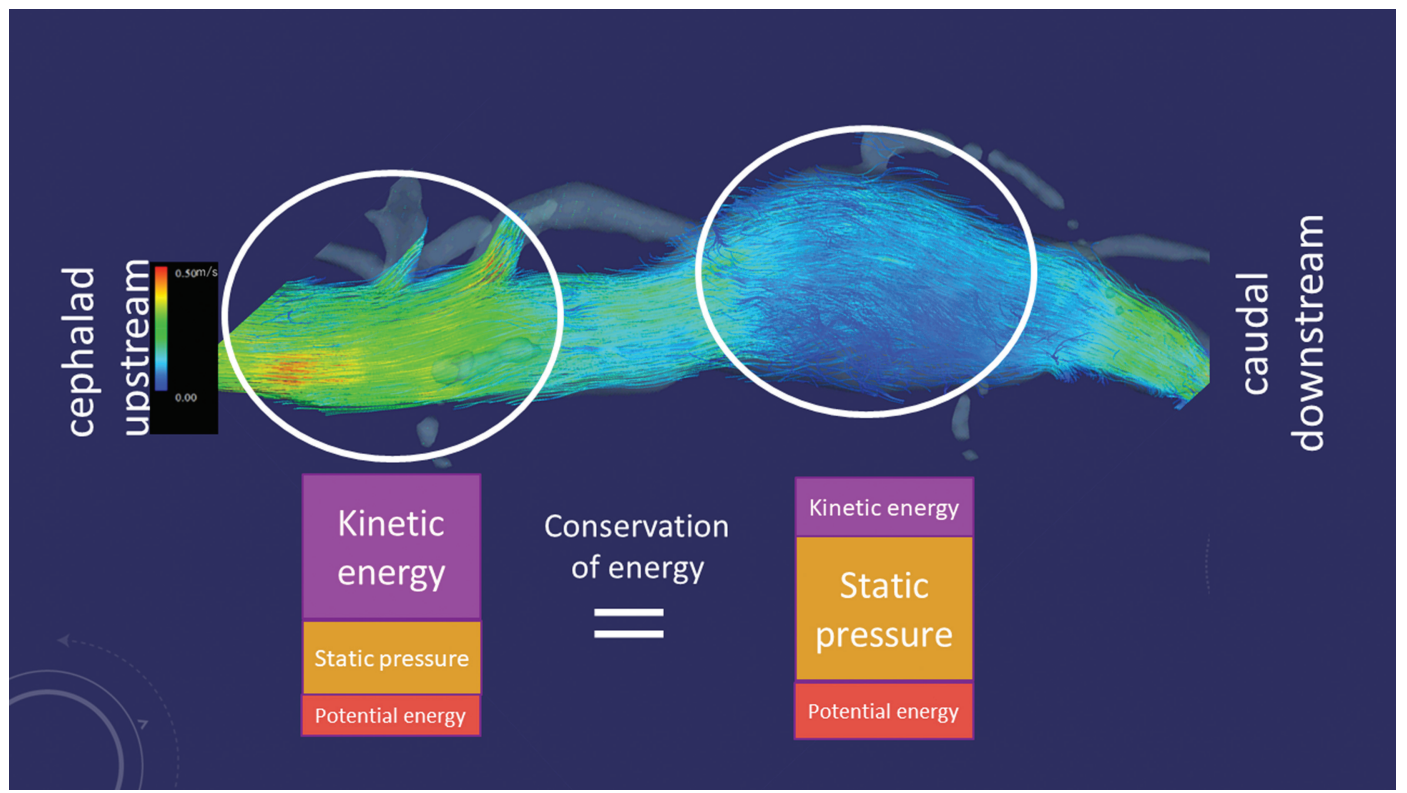

Fig. 7 Fluid energy is a combination of kinetic energy, static pressure, and potential energy. In the adjacent upstream and downstream aortic lumens, the law of conservation of energy holds. In the upstream non-expanding segment, the internal flow velocity is high; therefore, the kinetic energy is high and the static pressure is low (Intuitively, the higher the flow velocity, the higher the static pressure; however, in reality, the opposite is true). In contrast, the slower blood flow in the AAA loses kinetic energy; hence, the loss of kinetic energy in the AAA implies an increase in local static pressure. AAA, abdominal aortic aneurysm.

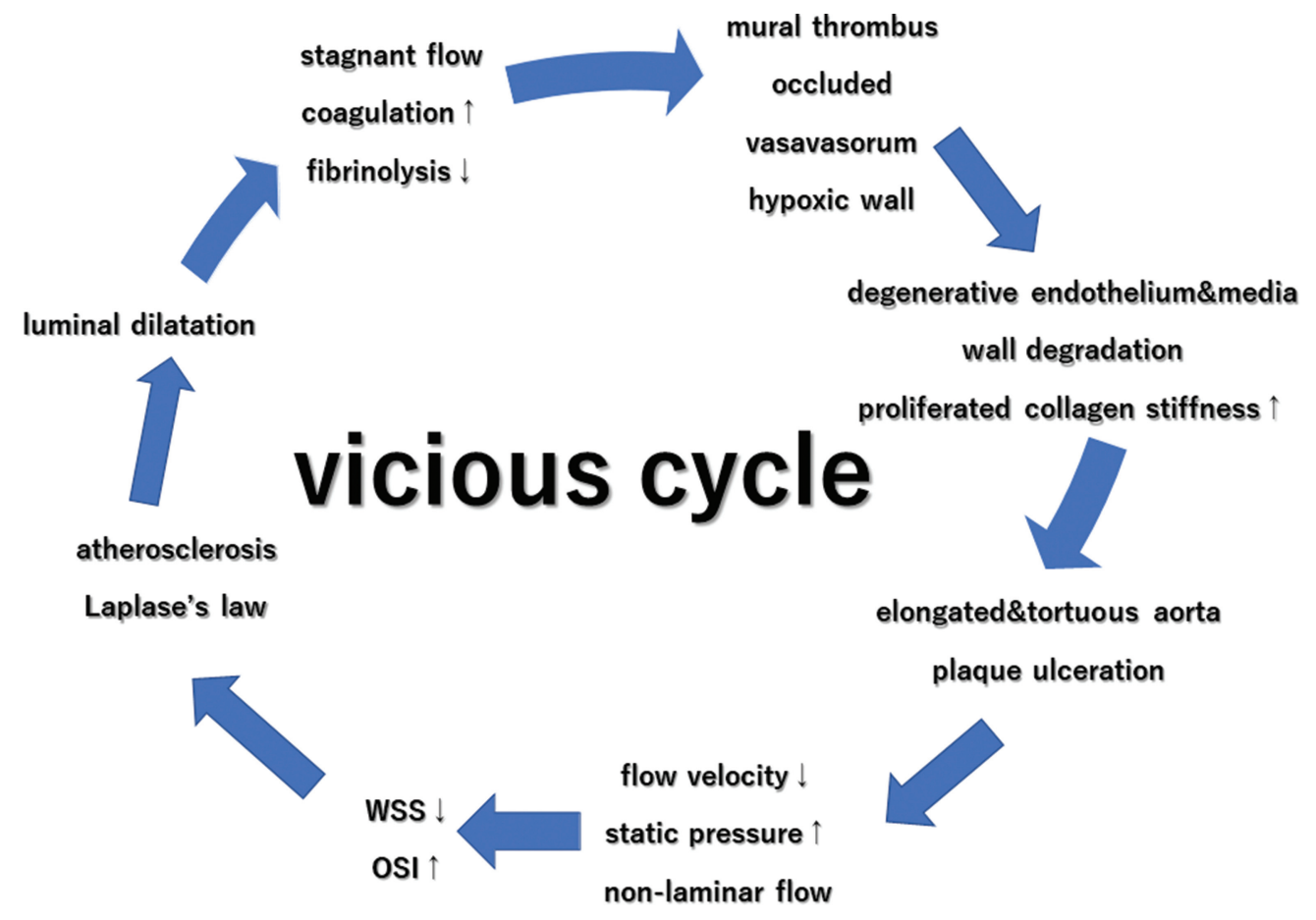

Fig. 8 Schematic of the vicious cycle in the development of the abdominal aortic aneurysm. In the flow-disturbed portion in the aorta, the flow velocity and WSS decrease, whereas the OSI increases, which continuously induce atherogenic changes in the aorta, thereby degrading the aortic wall integrity. The loss of kinetic energy also increases the local static pressure, as seen in Fig. 7 . The aortic wall begins to bulge under increased static pressure and degrade the aortic wall. Once the aorta starts to expand, the expansion is accelerated with less force. This phenomenon is known as Laplace's law. As the aorta expands, the dilation leads to more disturbing flow due to an inverse pressure gradient; consequently, the WSS decreases and OSI increases. At the same time, the low WSS and high OSI promote coagulation and hinder fibrinolysis. Together with stagnant blood flow and coagulation, a mural thrombus is induced. Thus, the vasavasorum is impeded. The oxygen diffusion from the blood is also lost, which further damages the wall and makes it unable to withstand the increase in static pressure, causing further dilation. OSI, oscillatory shear index; WSS, wall shear stress. 
a

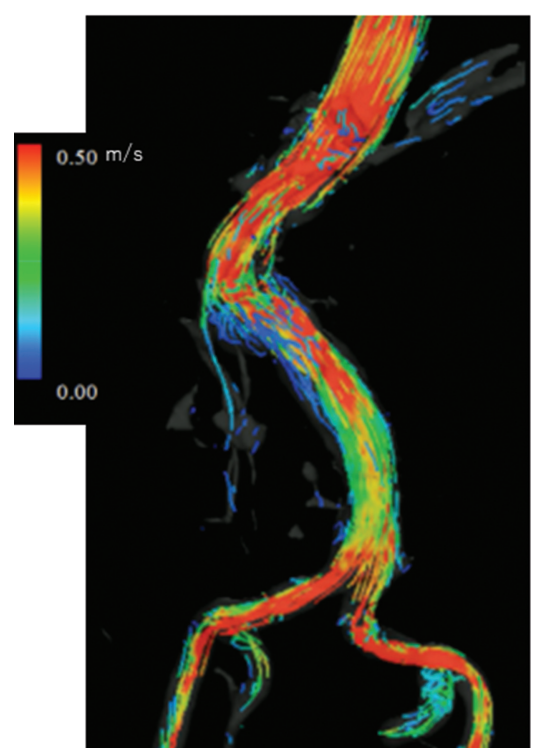

b

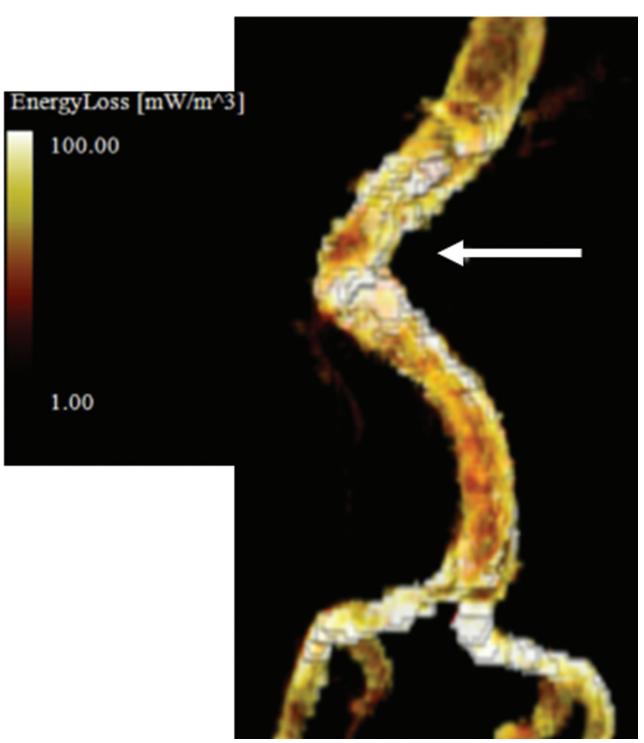

Fig. 9 The same AAA case as shown in Figs. 3 and 6 treated with EVAR a: Streamline image at systole showing increased flow velocity within the stent-graft. $\mathbf{b}$ : An EL image at systole showing increased EL caused by non-laminar flow due to kinked neck (arrow) and increased velocity due to reduced luminal volume of the stent-graft. EL, energy loss. AAA, abdominal aortic aneurysm; EL, energy loss; EVAR, endovascular aortic repair.
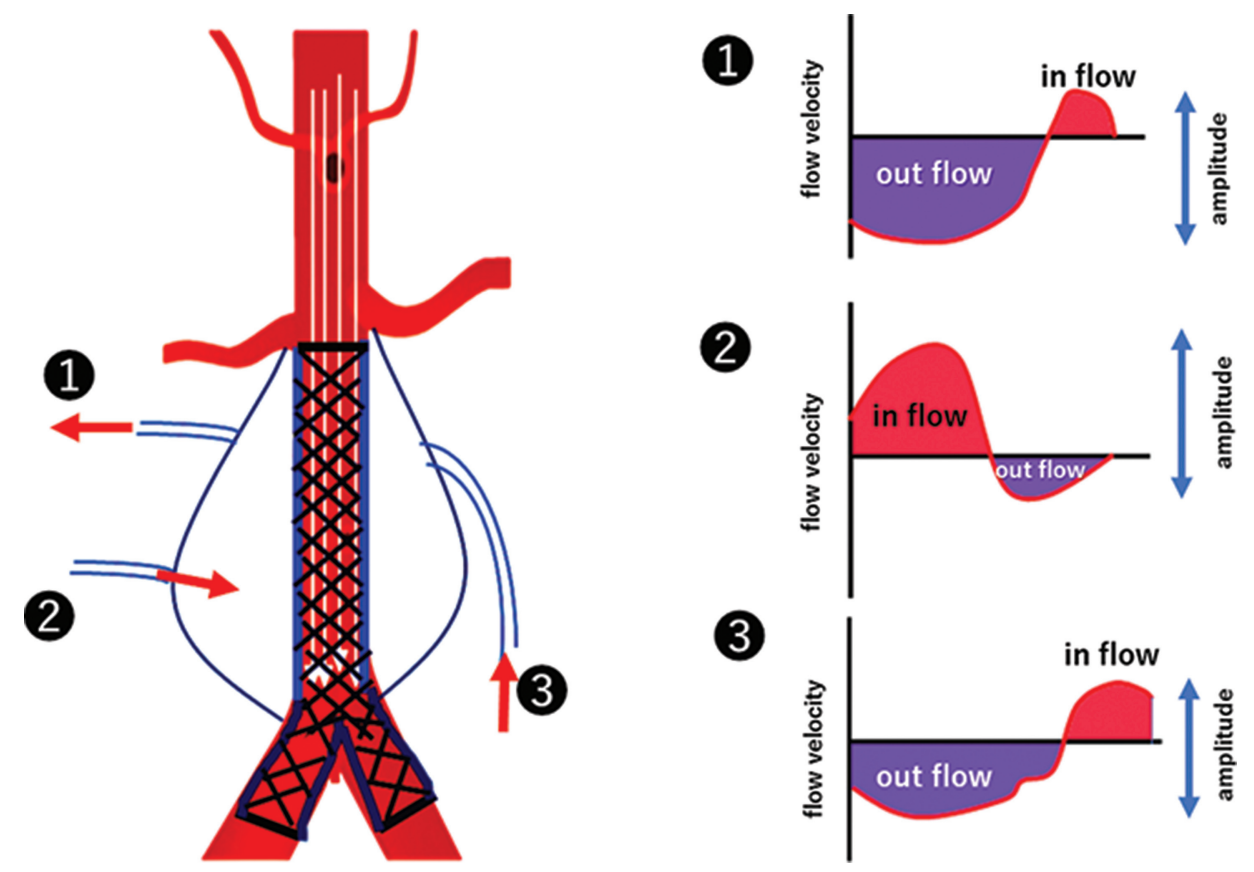

Fig. 10 Schematic showing an example of type II endoleak after endovascular stent-graft placement within the abdominal aortic aneurysm. In type II endoleak, there are several blood inflow or outflow channels to and from the aneurysmal sac via tributary arteries. 4D flow MRI can assess the blood flow for each tributary arteries, as well as the balance of the inflow and outflow velocities or volumes. The flow velocity and direction in the multiple tributary arteries branching from the aneurysm are not uniform. In this example, all the flow in the branches are biphasic; however, artery \#1 is outflow dominant, \#2 is inflow dominant, and \#3 is outflow dominant. The amplitude of the flow velocities is the highest for branch \#2 and lowest for branch \#3. 
a

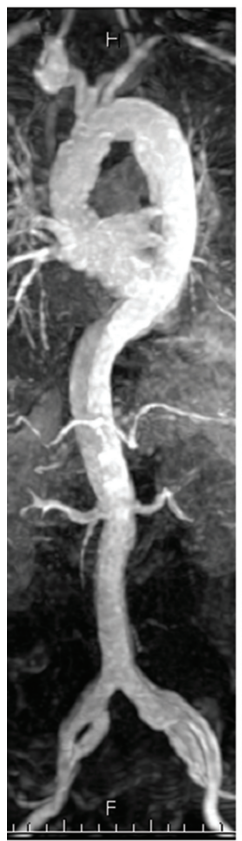

early b

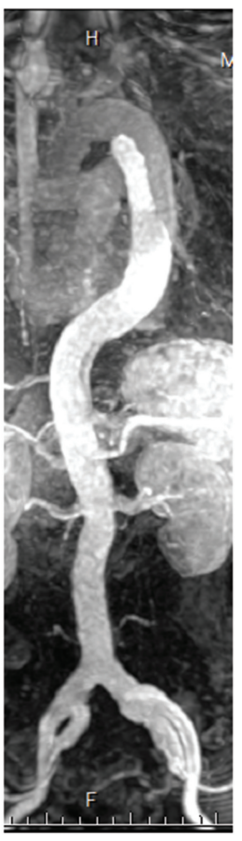

delayed

\section{CE MR Angiography}

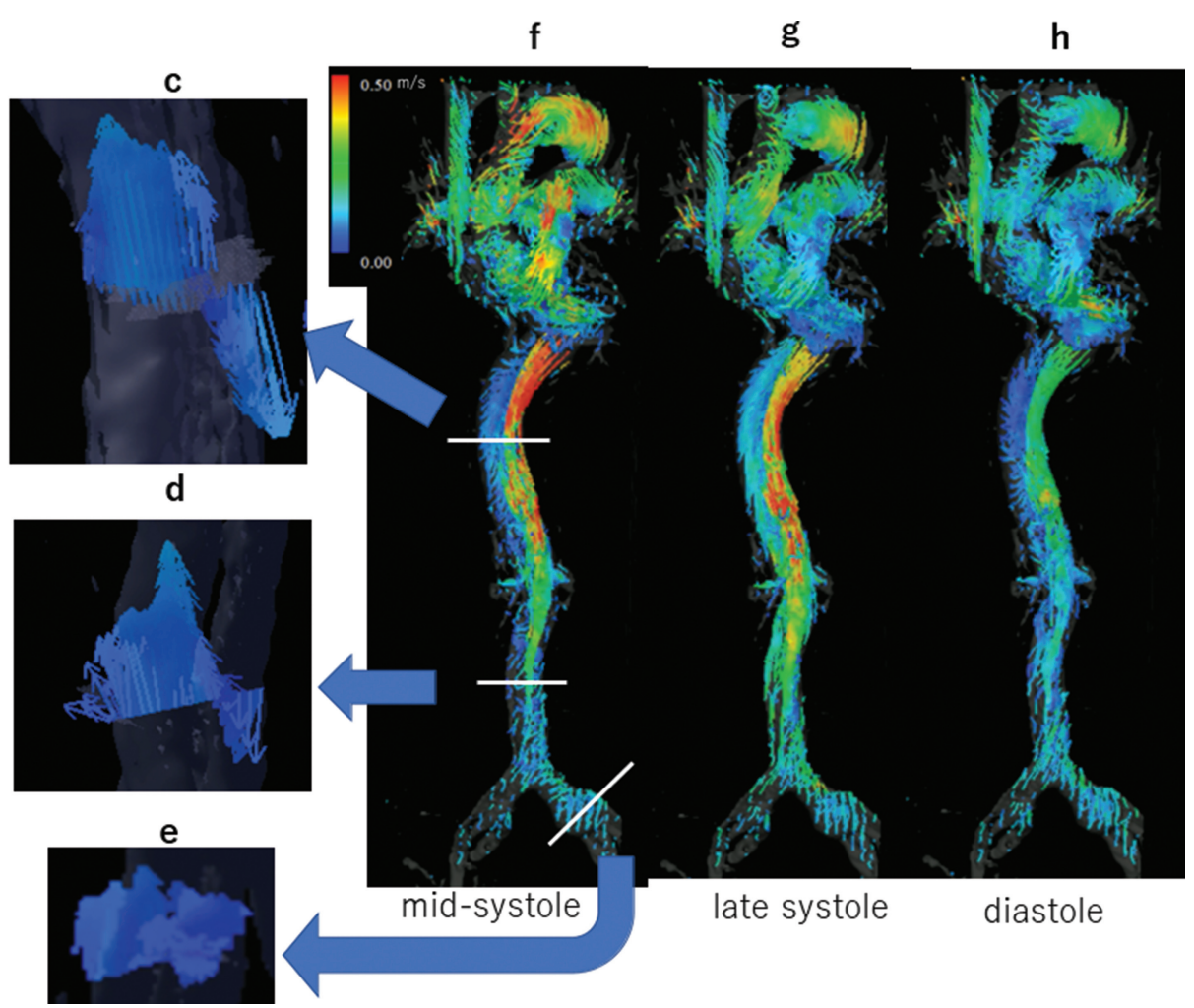

Fig. 11 A female patient in her 70s with Stanford type A aortic dissection after graft replacement at the ascending aorta. Her true and false lumen were hemodynamically assessed using 4D flow MRI. a and b Multi-phase-contrast-enhanced MR angiography indicates the portion of the true lumen and false lumen; however, detailed hemodynamics such as flow directions cannot be determined from the images. a: In an early phase, the portion of high enhancement (left side of the aorta) reflects the true lumen. b: In a delayed phase, the enhanced portion (primarily the right side of the aorta) is a false lumen. c-e Velocity vector map at mid-systole created at the three sections shown on the streamline image postprocessed using 4D flow MRI (f). Note that in the false lumen, the blood flow is reversed at systole, which is opposite in direction to the true lumen. This opposite flow direction in the false lumen reflects the dominant inflow into the false lumen from the reentry channel in the left common iliac artery. $\mathbf{f}-\mathbf{h}$ Streamline images postprocessed using 4D flow MRI acquired at (f) mid-systole, (g) latesystole, and (h) diastole. High-velocity flow is indicated in red.

direction of the various tributary arteries branching from the AAA are not uniform. There are branches with blood flowing into or out of the AAA. Simultaneous measurement of these arteries fascillitates the balance of the blood flow volume to the AAA over one cardiac cycle (Fig. 10). Katahashi et al. reported that comprehensive analysis of type II endoleak vessels per sac showed that the measured amplitude of the blood flow velocity in the tributary arteries was significantly higher in the aneurysmal sac of the expanding-group than in the non-expanding group. ${ }^{28}$

However, when interpreting the data of 4D flow MRI, one should be aware that it can only measure flows that repeat with the cardiac cycle. Thus, sporadic blood flow related to respiratory motion or changes in aneurysmal sac pressure may not be properly reflected. The other concern when measuring the flow velocity of tiny vessels is the bias that arises from the limited spatial resolution of the 4D flow MRI. In a study using a static flow phantom with a diameter of 3 $\mathrm{mm}$, the measurement error of 4D flow MRI in terms of spatially averaged velocity was less than $20 \%$ for a submillimeter pixel size. However, it was greater than $40 \%$ for a pixel size of $1.0 \mathrm{~mm} .{ }^{29}$ As the standard spatial resolution for current 4D flow MRI is around $1.0 \mathrm{~mm}$, errors of $20 \%-40 \%$ should be taken into account in the flowmetry of tiny vessels.

\section{Aortic Dissection and Organ Malperfusion}

As most abdominal aortic dissections are extensions of the pathology of thoracic aorta, few abdominal aortic dissections are confined to the abdominal aorta. Stanford type A, involving the ascending aorta, requires emergency treatment; therefore, it may not be suitable for assessment with 4D flow MRI. Although type B dissections are usually treated conservatively or placed on stand-by for elective surgery, 4D flow MRI may be considered. ${ }^{30}$ In subacute or chronic aortic dissection, it has become clear that pseudolumen patency (Fig. 11) and malperfusion of the organs (impaired branch perfusion) strongly affect treatment outcomes. There are 
reports of flow velocity measurements in the true and false lumen using 4D flow MRI. ${ }^{31-34}$ Allen et al. reported that 4D flow MRI could depict fenestration of the intimal flap better than CT angiography. ${ }^{35}$ Other investigators have reported that the flow velocity in the true lumen is higher than that of the false lumen. A more significant absolute value of the flow velocity in the false lumen has a greater tendency to open the false lumen. ${ }^{36}$ Concerning the qualitative flow patterns within the false lumen, Francois et al. noticed a retrograde or helical flow within the false lumen. ${ }^{37}$

Hemodynamic assessments of the false lumen using 4D flow MRI may contribute to the risk stratification of the aortic dissection. Several hemodynamic factors, including higher flow volume, higher flow velocity, retrograde flow direction, and turbulence formation in the false lumen, are reported to be related to the outcomes of the affected aorta. ${ }^{38-40}$ Burris et al. has proposed an interesting concept of "ejection fraction of the false lumen," which describes the proportion of exiting flow from the false lumen at diastole measured at the primary entry tear. ${ }^{41}$ They reported that the index revealed a moderate-to-strong correlation with aortic growth rate. As the outflow from the false lumen overcomes the pressure of the true lumen, the index may reflect the elevated pressure of the false lumen. Standardized imaging protocol measuring local flow patterns of aortic dissections may provide valuable insights into individual patients and might help with their clinical management and assessment of prognosis. ${ }^{42,43}$

As for the estimation of malperfusion of the visceral organs affected by aortic dissection, there is no coherent report to date. To verify the utility of 4D flow MRI in assessing aortic dissections, large-scale multicenter studies using 4D flow MRI are anticipated.

\section{Use of 4D Flow MRI in Preventive Medicine}

As discussed above, a certain steady flow velocity level is required to maintain the vascular wall integrity. Given that the 4D flow MRI can be used to detect abnormalities in the WSS non-invasively, it can also be used to predict future vascular lesions and it may contribute toward the search for and early treatment of such vascular lesions (or even their prevention).

The easiest way to achieve increased abdominal WSS is performing lowerlimb exercises. ${ }^{44,45}$ Exercising the lower extremities increases the blood supply to the muscles of the lower extremities, which increases the blood flow velocity and WSS of the pelvic arteries and upstream abdominal aorta. Using a 2D phase-contrast method, Taylor et al. measured the WSS in the abdominal aorta before and after lowerlimb exercise stress. They reported a significant increase in the WSS from $1.3 \pm 0.8 \mathrm{~Pa}$ at rest to $5.2 \pm 1.3 \mathrm{~Pa}$ during exercise. The OSI, which reflects the WSS fluctuation, reduced to zero after exercise. They evaluated normal subjects and patients with intermittent claudication, and reported that the latter showed lower WSS upon effort.

\section{Future Prospects}

As MRI is a non-invasive and ionizing-radiation-free technique, 4D flow MRI can be repeated as many times as required. Thus, it fascilitates comprehensive, retrospective, and quantitative assessments of pathological hemodynamic changes caused by vascular diseases, as well as physiological hemodynamic changes after exercise or drug loading. Therefore, it is expected to become an optimal method for diagnosis, drug discovery, and preventive medicine in the future.

\section{Conflicts of Interest}

The corresponding author Yasuo Takehara is an endowed chair of a department that is financially supported by a private company; however, this status is irrelevant to the contents of the paper.

\section{References}

1. Ando J, Komatsuda T, Ishikawa C, Kamiya A. Fluid shear stress enhanced DNA synthesis in cultured endothelial cells during repair of mechanical denudation. Biorheology 1990; 27:675-684.

2. Ando J, Yamamoto K. Hemodynamic forces, endothelial mechanotransduction, and vascular diseases. Magn Reson Med Sci 2021 May 22. [Epub ahead of print]

3. Malek AM, Alper SL, Izumo S. Hemodynamic shear stress and its role in atherosclerosis. JAMA 1999; 282:2035-2042.

4. Malek AM, Izumo S. Control of endothelial cell gene expression by flow. J Biomech 1995; 28:1515-1528.

5. Rogers WJ, Hu YL, Coast D, et al. Age-associated changes in regional aortic pulse wave velocity. J Am Coll Cardiol 2001; 38:1123-1129.

6. Dietenbeck T, Craiem D, Rosenbaum D, et al. 3D aortic morphology and stiffness in MRI using semi-automated cylindrical active surface provides optimized description of the vascular effects of aging and hypertension. Comput Biol Med 2018; 103:101-108.

7. Hwang J, Ing MH, Salazar A, et al. Pulsatile versus oscillatory shear stress regulates NADPH oxidase subunit expression: implication for native LDL oxidation. Circ Res 2003; 93:1225-1232.

8. Tsuji T, Suzuki J, Shimamoto R, et al. Vector analysis of the wall shear rate at the human aortoiliac bifurcation using cine MR velocity mapping. AJR Am J Roentgenol 2002; 178:995-999.

9. Wehrum T, Dragonu I, Strecker C, et al. Influence of pulse wave velocity on atherosclerosis and blood flow reversal in the aorta: A 4-dimensional flow magnetic resonance imaging study in acute stroke patients and matched controls. J Thorac Imaging 2022; 37:42-48.

10. Winter P, Andelovic K, Kampf T, et al. Simultaneous measurements of 3D wall shear stress and pulse wave velocity in the murine aortic arch. J Cardiovasc Magn Reson 2021; 23:34. 
11. Hermann I, Shchetynska-Marinova T, Amendt K, et al. First experiences of local pulse wave velocity measurements in $4 \mathrm{D}$ MRI in focally stented femoropopliteal arteries. Vasa 2021; 50:468-474.

12. Honda $\mathrm{T}$, Itatani $\mathrm{K}$, Takanashi $\mathrm{M}$, et al. Quantitative evaluation of hemodynamics in the Fontan circulation: a cross-sectional study measuring energy loss in vivo. Pediatr Cardiol 2014; 35:361-367.

13. Itatani $\mathrm{K}$, Okada $\mathrm{T}$, Uejima $\mathrm{T}$, et al. Intraventricular flow velocity vector visualization based on the continuity equation and measurements of vorticity and wall shear stress. Jpn J Appl Phys 2013; 52:07HF16.

14. Itatani K, Sekine T, Maeda $Y$, et al. Hemodynamic parameters for 4D Flow MRI: Mathematical definition and clinical applications. Magn Reson Med Sci 2021 February 16. [Epub ahead of print]

15. Iwata K, Sekine T, Tanaka I, Ando T, Orita E. Turbulent kinetic energy is different from viscous energy loss. Radiographics 2020; 40:2142-2144.

16. Elbaz MSM, Scott MB, Barker AJ, et al. Four-dimensional virtual catheter: Noninvasive assessment of intra-aortic hemodynamics in bicuspid aortic valve disease. Radiology 2019; 293:541-550.

17. Oyama-Manabe N, Aikawa T, Tsuneta S, Manabe O. Clinical applications of $4 \mathrm{D}$ flow MR imaging in aortic valvular and congenital heart disease. Magn Reson Med Sci 2021 Jun 25. [Epub ahead of print]

18. Ha H, Kim GB, Kweon J, et al. Turbulent kinetic energy measurement using phase contrast mri for estimating the post-stenotic pressure drop: In vitro validation and clinical application. PLoS One 2016; 11:e0151540.

19. Learoyd BM, Taylor MG. Alterations with age in the viscoelastic properties of human arterial walls. Circ Res 1966; 18:278-292.

20. Sehgel NL, Sun Z, Hong Z, et al. Augmented vascular smooth muscle cell stiffness and adhesion when hypertension is superimposed on aging. Hypertension 2015; 65:370-377.

21. Sehgel NL, Vatner SF, Meininger GA. "Smooth muscle cell stiffness syndrome"-Revisiting the structural basis of arterial stiffness. Front Physiol 2015; 6:335.

22. Sugawara J, Hayashi K, Yokoi T, Tanaka H. Age-associated elongation of the ascending aorta in adults. JACC Cardiovasc Imaging 2008; 1:739-748.

23. Brewster DC, Cronenwett JL, Hallett JW Jr., Johnston KW, Krupski WC, Matsumura JS. Guidelines for the treatment of abdominal aortic aneurysms. Report of a subcommittee of the Joint Council of the American Association for Vascular Surgery and Society for Vascular Surgery. J Vasc Surg 2003; 37:1106-1117.

24. Prager M, Claeys L, Fugl A, et al. Abdominal aortic aneurysm: surgery, indications, technique, outcome. Acta Med Austriaca 1997; 24:10-14.

25. Takehara $\mathrm{Y}$, Isoda $\mathrm{H}$, Takahashi $\mathrm{M}$, et al. Abnormal flow dynamics result in low wall shear stress and high oscillatory shear index in abdominal aortic dilatation: Initial in vivo assessment with 4D-flow MRI. Magn Reson Med Sci 2020; 19:235-246.

26. Webb G. Magnetohydrodynamics and fluid dynamics: Action principles and conservation laws. Cham: Springer International Pulishing 2018.
27. Anton R, Chen CY, Hung MY, Finol EA, Pekkan K. Experimental and computational investigation of the patient-specific abdominal aortic aneurysm pressure field. Comput Methods Biomech Biomed Engin 2015; 18:981-992.

28. Katahashi K, Sano M, Takehara Y, Inuzuka K, Sugiyama M, Alley MT, et al. Flow dynamics of type II endoleaks can determine sac expansion after endovascular aneurysm repair using four-dimensional flow-sensitive magnetic resonance imaging analysis. J Vasc Surg 2019; 70:107-116.e1.

29. Fukuyama $\mathrm{A}$, Isoda $\mathrm{H}$, Morita $\mathrm{K}$, et al. Influence of spatial resolution in three-dimensional cine phase contrast magnetic resonance imaging on the accuracy of hemodynamic analysis. Magn Reson Med Sci 2017; 16:311-316.

30. Amano Y, Sekine T, Suzuki Y, Tanaka K, Takagi R, Kumita S. Time-resolved three-dimensional magnetic resonance velocity mapping of chronic thoracic aortic dissection: a preliminary investigation. Magn Reson Med Sci 2011; 10:93-99.

31. Chen CW, Fang YF, Tseng YH, et al. Before and after endovascular aortic repair in the same patients with aortic dissection: A cohort study of four-dimensional phase-contrast magnetic resonance imaging. Diagnostics (Basel) 2021; 11:1912.

32. Chen CW, Tseng YH, Lin CC, et al. Aortic dissection assessment by $4 \mathrm{D}$ phase-contrast MRI with hemodynamic parameters: the impact of stent type. Quant Imaging Med Surg 2021; 11:490-501.

33. Liu D, Fan Z, Li Y, et al. Quantitative study of abdominal blood flow patterns in patients with aortic dissection by 4 dimensional flow MRI. Sci Rep 2018; 8:9111.

34. Saitta S, Guo B, Pirola S, et al. Qualitative and quantitative assessments of blood flow on tears in type B aortic dissection with different morphologies. Front Bioeng Biotechnol 2021; 9:742985.

35. Allen BD, Aouad PJ, Burris NS, Rahsepar AA, Jarvis KB, Francois CJ, et al. Detection and hemodynamic evaluation of flap fenestrations in type B aortic dissection with 4D flow MRI: Comparison with conventional MRI and CTA. Radiol Cardiothorac Imaging. 2019; 1:e180009.

36. Inoue $\mathrm{T}$, Watanabe $\mathrm{S}$, Sakurada $\mathrm{H}$, et al. Evaluation of flow volume and flow patterns in the patent false lumen of chronic aortic dissections using velocity-encoded cine magnetic resonance imaging. Jpn Circ J 2000; 64:760-764.

37. Francois CJ, Markl M, Schiebler ML, et al. Four-dimensional, flow-sensitive magnetic resonance imaging of blood flow patterns in thoracic aortic dissections. J Thorac Cardiovasc Surg 2013; 145:1359-1366.

38. Clough RE, Waltham M, Giese D, Taylor PR, Schaeffter T. A new imaging method for assessment of aortic dissection using four-dimensional phase contrast magnetic resonance imaging. J Vasc Surg 2012; 55:914-923.

39. Takahashi K, Sekine T, Ando T, Ishii Y, Kumita S. Utility of $4 \mathrm{D}$ flow MRI in thoracic aortic diseases: A Literature Review of Clinical Applications and Current Evidence. Magn Reson Med Sci 2021 Sep 8. [Epub ahead of print]

40. Takahashi K, Sekine T, Miyagi Y, et al. Four-dimensional flow analysis reveals mechanism and impact of turbulent flow in the dissected aorta. Eur J Cardiothorac Surg 2021; 60:1064-1072. 
41. Burris NS, Nordsletten DA, Sotelo JA, et al. False lumen ejection fraction predicts growth in type B aortic dissection: preliminary results. Eur J Cardiothorac Surg 2020; 57:896-903.

42. Zilber ZA, Boddu A, Malaisrie SC, et al. noninvasive morphologic and hemodynamic evaluation of type $\mathrm{B}$ aortic dissection: State of the art and future perspectives. Radiol Cardiothorac Imaging. 2021; 3:e200456.

43. Rudenick PA, Segers P, Pineda V, et al. False lumen flow patterns and their relation with morphological and biomechanical characteristics of chronic aortic dissections. Computational model compared with magnetic resonance imaging measurements. PLoS One 2017; 12:e0170888.
44. Edwards DG, Schofield RS, Lennon SL, Pierce GL, Nichols WW, Braith RW. Effect of exercise training on endothelial function in men with coronary artery disease. Am J Cardiol 2004; 93:617-620.

45. Edwards DG, Schofield RS, Magyari PM, Nichols WW, Braith RW. Effect of exercise training on central aortic pressure wave reflection in coronary artery disease. Am J Hypertens 2004; 17:540-543.

46. Takehara $\mathrm{Y}$, Isoda $\mathrm{H}$, Takahashi $\mathrm{M}$, et al. Abnormal flow dynamics result in low wall shear stress and high oscillatory shear index in abdominal aortic dilation: Initial in vivo assessment with 4D-flow MRI. Magn Reson Med Sci 2020; 19:235246. 